\title{
Niños testigos de violencia de género. Víctimas directas
}

\section{Evaluna Pereyra Eufrasio ${ }^{a}$}

RESUMEN: La violencia de género supone un problema que afecta de manera significativa los vértices de la estructura social. Uno de los sectores poblacionales que sufren el mayor impacto son los niños expuestos, sea en calidad de víctima o testigo, a contextos que atentan contra la integridad de las mujeres, en especial si se trata de sus madres o principales cuidadoras. Sin embargo, la legislación mexicana aún no reconoce al menor que sufrió un agravio de este tipo como una víctima directa. El presente documento apela a recuperar propuestas de diversos estudios psicológicos, que demuestran cómo la exposición de los niños a eventos traumáticos, incluso en calidad de testigos, tiene repercusiones negativas en su desarrollo físico, cognitivo y emocional de la misma magnitud que un abuso directo.

Palabras clave: Violencia de género; abuso infantil; teoría del apego; padres y cuidadores; legislación mexicana.

ABSTRACT: Gender violence suppose a problem that affects in a significative manner the apexes of the social structure. One of the population groups who suffers the highest impact are children exposed, be as a victim or a witness, to a context that attempts against the women's integrity, especially mothers or main caretakers. However, Mexican legislation has not recognized yet the minor who has suffered grievance of this kind as the way a direct victim has. This document appeals to recover proposals from various psychological studies that show that the exposition of children to traumatic events as of witnesses has negative repercussions in their physical, cognitive, and emotional with the same magnitude that a direct abuse.

Keywords: Gender Violence; Children Abuse; Parents and Caretakers; Attachment Theory; Mexican Legislation.

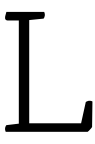

a legislación de atención a víctimas en el estado de Veracruz, contempla como víctima directa a la persona que haya sufrido algún daño o menoscabo físico, mental, emocional, así como cualquier acto que atente contra sus derechos jurídicos; por otra parte,

\footnotetext{
a Estudiante de la licenciatura en Lengua y Literatura Hispánicas, Universidad Veracruzana. Colaboradora del Observatorio Universitario de Violencias contra las Mujeres. Contacto: evaluna pe@hotmail.com.
} 


\section{Nota Breve - Observatorio Universitario de Violencias Contra las Mujeres}

conceptualiza como víctima indirecta a aquellas personas que mantienen una relación familiar o de cercanía con el agraviado. Acorde a esta legislación, el Reglamento de la Ley de Acceso de las mujeres a una vida libre de violencia para el estado de Veracruz de Ignacio de la Llave considera que los menores, cuyas madres fueron asesinadas por razones de género, deben ser atendidos en calidad de víctima indirecta; no obstante, parece ser que esta clasificación supone una contradicción en sí misma, ya que la violencia de género -incluida aquella que culmina en el feminicidio- hacia las mujeres que son madres repercute y agravia de forma directa a quienes dependen de ella.

Por su parte el Manual de atención a niños y niñas víctimas de violencia de género en el ámbito familiar (2008) de la asociación internacional Save the children mantiene que los infantes deben ser considerados víctimas de la violencia de género, ya que ésta atenta contra sus derechos humanos, así como contra garantías particulares de su condición previstas en La Declaración de los Derechos del Niño, documento internacional que, desde su promulgación en 1959, busca proveer a los menores de la seguridad y estabilidad que permitan su desarrollo íntegro; un contexto en el cual el infante está expuesto a la violencia, sea en calidad de víctima o testigo, supone uno de los principales impedimentos.

Para los menores, el seno familiar desempeña un papel de crucial importancia al ser el primer contexto que los cobija y ser aquel en el que mayor tiempo se encuentran; dentro del mismo los progenitores cumplen el rol de "cuidadores" o personas encargadas de proporcionar al niño el ambiente y las herramientas necesarias para el desarrollo de sus facultades mentales, emocionales y sociales, es decir proveen de la seguridad, confianza y regulación para posibilitar la libre expresión del niño, de la misma manera deben brindar protección ante los agentes de riesgo los cuales incluyen enfermedades, accidentes y agresores sociales. En los casos de violencia intrafamiliar los pequeños experimentan una constante amenaza de primera mano: un miembro cercano, por lo general son los padres o cuidadores principales (abuelos, tíos), es la misma persona que le genera daño físico o psicológico.

Cabe aquí preguntarse ¿Cuál es la consecuencia en aquellos contextos en los que el niño identifica a su progenitor como cuidador y al mismo como el agente amenazante? Según la teoría del apego ${ }^{1}$ desarrollada por John Bowlby (1969), con las aportaciones de Mary

\footnotetext{
${ }^{1}$ Teoría de corte psicológico, evolutivo y etiológico, postulada formalmente en el libro Attachment (Bowlby, 1969); su gestación se remonta a los primeros postulados de John Bowlby publicados en 1951, resultado de estudios en niños víctimas de la Segunda Guerra Mundial. Esta teoría propone que el ser humano es un animal social, cuya adaptación evolutiva es el desarrollo de apegos, los cuales deben entenderse como vínculos afectivos entre un individuo y una figura de apego (o cuidador). La postura de Bowlby, desarrollada posteriormente Ainsworth, 
Nota Breve - Observatorio Universitario de Violencias Contra las Mujeres

Ainsworth (1967) y ampliada por Main y Solomon (1986) el menor genera un apego desorganizado. Los pequeños aún no tienen la capacidad para sobrevivir sin la presencia de su fuente principal de cuidados, por ende deben soportar los maltratos o negligencias, esto genera un importante desequilibrio en la salud mental del infante, pues se le brindan estímulos contradictorios; las consecuencias desprendidas del apego desorganizado son plurales: desregulación emocional, trastorno de ansiedad crónica, depresión constante, invalidación, sentimientos de culpa, autolesiones, mecanismos de defensa no efectivos como la disociación, siendo la más graves daños cerebrales a nivel fisiológico y por su puesto el latente riesgo de suicidio.

La asociación de los cuidadores como agentes amenazantes puede darse en caso de que éstos inflijan menoscabo directo sobre el niño, o cuando el infante es testigo del daño, o amenaza de la integridad de otra persona, e incluso de otro ser². En este último caso existen dos factores cruciales, el primero es la cercanía que se mantiene con el afectado, y el segundo es la edad del testigo, entre menor sea, mayor es el potencial trauma, pues los infantes durante los primeros años de vida desarrollan mecanismos de integración y construcción de un sentido del yo3; los niños pequeños no distinguen entre su persona y la del otro, razón por la cual pueden percibir el daño hacia alguien más como si fuesen ellos mismos el agraviado, eso

\footnotetext{
plantea como esenciales los primeros tres años de vida, ya que en éstos el infante genera los primeros modelos de experiencia que lo determinarán en su vida futura. M. Ainsworth propone algunos tipos de patrones: apego seguro, apego inseguro-evitativo, apego inseguro-ambivalente y el apego desorganizado, integrado por Main y Salomon (1986).

${ }^{2}$ En la categoría se puede englobar la violencia dirigida a un animal o a objetos personales del infante como lo son elementos de juego antropomorfizados: muñecas, peluches, figuras de acción, así como productos audiovisuales, como se ha señalado el trauma es de naturaleza subjetiva y depende de los mecanismos con los que cuente el individuo.

${ }^{3}$ Entre estos se pueden incluir la mentalización, que es la facultad de poder diferenciar entre la realidad interna y externa, principalmente implica el reconocer los juicios ajenos y propios. "En su interacción el niño comprenderá el comportamiento de los otros, siempre y cuando adquiera en su entorno un sentimiento de seguridad y confianza." (María Turaglio, 2013, p. 6). La teoría de la mente que refiere a la capacidad de atribuir pensamientos, intenciones y emociones al otro. El desarrollo de neuronas espejo, término dado a un grupo de neuronas localizadas en la circunvolución frontal inferior y la corteza parietal inferior, involucradas en la imitación de los comportamientos de los otros. Así como la capacidad del individuo para la integración de experiencias, proceso en el que intervienen mecanismos que posibilitan la vinculación y diferenciación de eventos a través del tiempo -synthesis-, así como aquellos que le permiten al individuo darse cuenta/ hacer real una experiencia desde un sentido del yo "esto me está pasando a mí, pienso y me siento así respecto a" y a situarlas en una línea temporal -realization-.
} 
explicaría porque niños testigos de un evento amenazante presentan los mismos síntomas que los niños víctimas de un abuso.

Antes de continuar se debe contemplar que, en nuestra estructura sociocultural, ${ }^{4}$ la relación que se establece entre los menores y su madre supone uno de los vínculos más significativos para el desarrollo del menor durante las primeras etapas, pues son las mujeres quienes desempeñan por antonomasia el papel de cuidador principal. Cuando la madre del menor sufre agresiones por parte de cualquier otro de los miembros de la familia —aunque por lo regular el agresor es el cónyuge, es decir padre-padrastro de los niños-el infante aun cuando no sea atacado padece respuestas significativas de estrés, a las que se aúna el hecho de la poca capacidad de distanciamiento del otro o mentalización.

El niño es víctima del mismo maltrato que la madre, e incluso puede sentir más temor y ansiedad por su incapacidad de defensa, en la psique del infante la muerte de su madre significa su muerte inminente. Además, las conductas de los cuidadores proporcionan modelos de acción que el infante adquiere y desarrolla, e incluso muchas veces introyecta; cuestión que en el futuro puede llevarlo a ser víctima o victimario.

Los infantes testigos de violencia son contenedores de una experiencia emocional súbita, significan una población vulnerable cuyos derechos humanos son violados, y que presentan consecuencias a lo largo de toda su vida. Por esta razón la violencia que sufren los menores "víctimas indirectas" de la violencia de género debería ser atendida como casos independientes de abuso psicológico; es necesario que los infantes adquieran visibilidad como víctimas directas de un agravio a fin de que su atención y seguimiento suponga un motivo principal en las agendas de atención pública.

\section{Referencias}

Ainsworth, M. D. (1967). Infancy in Uganda: infant care and the growth of love. Baltimore: Johns Hopkins.

Bowlby, J. (1969). Attachment. Attachment and Loss. Vol. I. Londres: Hogarth.

Main M. y Solomon J. (1986). "Discovery of an Insecure-Disorganized/ Disoriented Attachment Pattern”. en Berry, T., Yogman M. W. ed. Affective Development in Infancy. pp. 95124.Norwood, New Jersey: Ablex.

\footnotetext{
${ }^{4}$ Cabe aclarar que la socialización de los infantes está determinada en gran medida por los factores culturales, la teoría del apego ha sido discutida desde una perspectiva cultural en Mesman, J., Sagi-Schwartz, A., Van IJzendoorn, M. H., (2008). 
Nota Breve - Observatorio Universitario de Violencias Contra las Mujeres

Mesman, J., Sagi-Schwartz, A., Van M. H. (2008) "Cross-Cultural Patterns of Attachment; Universal and Contextual Dimensions". en Cassidy, J., Shaver, P. R., ed. Handbook of Attachment: Theory, Research and Clinical Applications. Nueva York y Londres: Guilford Press. pp. 880-905.

Ley 259 de Víctimas para el estado de Veracruz de Ignacio de la Llave. Gaceta Oficial, Órgano del Gobierno del Estado de Veracruz de Ignacio de la Llave, 4 de abril de 2017.

María Turaglio, L. (2013). Mentalización en madres con trastorno límite de la personalidad y la autorregulación emocional de los niños. [Revalidación de título Universidad de Chile] Universidad Pontificia Comillas: Madrid.

Save the Children (2008). Manual de atención a niños y niñas víctimas de violencia de género en el ámbito familiar. Recuperado de https://bit.ly/34Noh3m.

Reglamento de Ley 235 de Acceso de las mujeres a una vida libre de violencia para el estado de Veracruz de Ignacio de la Llave. Extraordinario de la Gaceta Oficial del Estado de Veracruz, el viernes 26 de febrero de 2010. 